\title{
Asteroseismic tests for models of magnetically active close binaries with orbital period modulation
}

\author{
A. F. Lanza ${ }^{1}$ and M. Rodonò ${ }^{2}$ \\ 1 Osservatorio Astrofisico di Catania of the Istituto Nazionale di Astrofisica, Via Santa Sofia, 78, Città Universitaria, \\ 95123 Catania, Italy \\ 2 Dipartimento di Fisica e Astronomia dell’Università degli Studi, Via Santa Sofia, 78, Città Universitaria, 95123 Catania, Italy \\ e-mail: mrodono@ct.astro.it
}

Received 23 January 2002 / Accepted 24 April 2002

\begin{abstract}
Magnetic activity and orbital period modulation in close binaries are considered in order to identify possible asteroseismic tests for models that have been proposed to understand their relationship. These models are based on the variation of the gravitational quadrupole moment of the active star, as a consequence of the modification of its internal rotation and magnetic field along its activity cycle (Applegate 1992; Lanza et al. 1998a). An analysis based on the solar analogy shows that surface magnetic activity may produce shifts, splitting and broadening of the $p$-mode peaks in the oscillation spectrum of a star. Such effects make the direct detection of the internal structural changes predicted by the models very difficult. However, a time variation of the internal rotation can be detectable because the $p$-mode rotational splittings are proportional to the azimuthal quantum number $m$, whereas structural and surface effects controlled by the magnetic fields are $|m|$ dependent. Therefore, we propose a method that correlates the variation of the $a_{1}$ splitting coefficients, that sample the internal rotation, with the orbital period variation in order to test the predictions of the models. The RS CVn short-period systems, such as V 471 Tau, RT And or CG Cyg, are the most suitable objects for such a test because surface magnetic effects are likely to be less prominent than in classical or long-period RS CVn binaries.
\end{abstract}

Key words. stars: binaries: close - stars: binaries: eclipsing - stars: oscillations - stars: magnetic fields - stars: activity

\section{Introduction}

The long-term timing of eclipses in several classes of close binaries has shown that their orbital periods are not constant and often oscillate around a mean value with time scales from a few to several decades. The phenomenon is well documented in the Algols and RS Canum Venaticorum binaries for which cyclic variations of the orbital period $P$ of amplitude $\Delta P / P \sim$ $(1-3) \times 10^{-5}$ have been reported, with a typical value of the modulation cycle of $\approx 40-50 \mathrm{yr}$ (cf. Hall 1989). Recent studies suggest a connection between orbital period modulation and magnetic activity on the component stars, as reviewed by, e.g., Hall (1989), Applegate (1992) and Lanza \& Rodonò (1999).

Several mechanisms have been proposed to explain such a connection, but serious problems are encountered in reproducing the short time scale of the observed changes and, at the same time, to meet the constraints set by the stellar energy budget (see, e.g., Applegate 1992; Lanza et al. 1998a; Lanza \& Rodonò 1999). The only mechanism that appears viable was proposed by Applegate (1992) and it was based on

Send offprint requests to: A. F. Lanza,

e-mail: nlanza@ct.astro.it cyclic changes of the gravitational quadrupole moment of the magnetically active component. Applegate's model assumes that a small fraction of the internal angular momentum of the active component is cyclically exchanged between an inner and an outer convective shell due to a varying internal magnetic torque versus the activity cycle phase. This modifies the oblateness and the gravitational quadrupole moment of the active star, which oscillates around its mean value. When the quadrupole moment is maximum, the companion star feels a stronger gravitational acceleration and is then forced to move closer and faster along its orbit attaining the minimum orbital period. Conversely, when the quadrupole moment is minimum the orbital period attains a maximum value.

In principle, a test of Applegate's model is possible by using the variation of the total area of starspots, which can be adopted as a proxy for the magnetic field on the active component. Moreover, spots can also be used as tracers of the surface rotation. Rodonò et al. (1995), Lanza et al. (1998b), Donati (1999), Lanza et al. (2002) performed such tests for RS CVn, AR Lac, HR 1099 and RT Lac, respectively. They found that for RS CVn, AR Lac and RT Lac the orbital period cycles are about two times longer than the spot activity cycles, at variance 
with Applegate's model. Moreover, the predicted angular velocity variations are too large compared with the changes of the spot rotation rate in all of the studied binaries.

In an attempt to overcome such difficulties, Lanza et al. (1998a) and Lanza \& Rodonò (1999) improved Applegate's model by including the effects of a strong internal magnetic field on the star figure of equilibrium. This may explain the length of the orbital modulation cycles and reduce the amplitude of the angular velocity changes. However, the difficulties in reconciling the models with the observations may also arise from the use of spots as tracers of the rotation changes. As a matter of fact, the analysis of starspot longitude distributions in close binaries from long-term time series have shown the existence of rather persistent active longitudes, which can affect the capability of spots to probe surface rotation (e.g., Henry et al. 1995; Rodonò et al. 1995; Eaton et al. 1996; Oláh et al. 1997; Lanza et al. 1998b; Lanza et al. 2001). Therefore, an independent tool to measure the time variation of the rotation rate of the outer convection zone would be desirable to confirm the results based on starspots and to perform a more stringent test of the models that have been proposed to explain the orbital period changes of active binaries. Such a tool may be provided by the measurements of the frequencies of the $p$-modes in active stars, which may soon become possible by space observatories.

For the Sun, the availability of spatially resolved data allows us to measure the internal rotation and its possible time variation with high accuracy (e.g., Schou et al. 1998; Howe et al. 2000). For distant stars we shall be able to measure only the frequencies of low degree $(l=0-3)$ oscillation modes with different radial order $n$. The expected spectrum has a characteristic appearance that can be predicted by the asymptotic theory of stellar oscillations (see Tassoul 1980; Brown \& Gilliland 1994). Moreover, the frequency splittings due to rotation can be measured and used to get some hints on the internal rotation of solar-like stars.

In the present paper we focus on the possibility offered by asteroseismic techniques to test the proposed models for orbital period modulation in close binaries. It is important to note that magnetic activity affects solar $p$-mode frequencies also through surface effects. Therefore, it is important to estimate what could be the effects of surface activity on the asymptotic $p$-mode spectrum of an active star in order to see whether we can disentangle the signatures of processes occurring deep in the convection zone from near-surface perturbations.

\section{Surface activity and $p$-mode frequencies}

Solar magnetic activity produces a variable increase of $p$-mode frequencies that, for modes with frequency $v \sim 3000 \mu \mathrm{Hz}$, at cycle maximum and minimum differs by $\sim 0.45 \mu \mathrm{Hz}$, that is $\Delta v / v \sim 10^{-4}$. Not only the central frequency of each multiplet is changed, but also the splittings are modified by solar activity. Since the current observations can detect variations in modes with $l$ as large as 100-150, it is customary to fit each frequency in a multiplet $v_{n l m}$ with a Legendre polynomial series:

$$
v_{n l m}=v_{n l 0}+\sum_{j=1}^{j=N} a_{j}(n, l) L P_{j}\left(\frac{m}{L}\right)
$$

where $n, l, m$ are the angular descriptors of the oscillation, $P$ is the Legendre polynomial and $L=l$ or $[l(l+1)]^{1 / 2}$ depending on the choice in the data reduction procedure; $N$ usually ranges from 5-6 to 30-35, depending on the maximum $l$ and the data accuracy (cf. Howe et al. 1999).

The lack of a detailed theoretical model of the interaction between magnetic activity and $p$-modes does not allow us to make definite predictions on the $p$-mode frequency behaviour in very active stars. However, we may obtain some hints by scaling the simple model proposed by Goldreich et al. (1991) to the stellar case. Their Eq. (20) can be scaled by assuming that $\rho(R) H \sim$ constant, where $\rho(R)$ is the density and $H$ the pressure scale height at the photosphere, and the mode masses $M_{\omega}$ are comparable to those of the solar modes. This yields the following relative perturbation of the low-degree $p$-mode frequency with $n \simeq 19-21$ (corresponding to the maximum power in the solar spectrum):

$\frac{\Delta v}{v} \sim 1.0 \times 10^{-2} f\left(\frac{R}{R_{\odot}}\right)^{3}\left(\frac{M}{M_{\odot}}\right)^{-1}\left(\frac{T_{\mathrm{eff}}}{6000}\right)$,

where $0 \leq f \leq 1$ is the filling factor of the magnetic field, $T_{\text {eff }}$ is the effective temperature in Kelvin and $R$ and $M$ are the radius and mass of the star, respectively. The photospheric filling factors of very active stars in close binaries are typically one or two orders of magnitude higher than in the solar case, and the field strength in the photospheric flux tubes can be assumed to be roughly in equipartition with the gas pressure (e.g., Saar 1996). Therefore, the expected $p$-mode frequency perturbations are correspondingly greater. Specifically, we may estimate the frequency shifts for $p$-modes of radial order $n=20$ in the case of the K2 V secondary of V471 Tauri, and of the K0 subgiant secondary of AR Lacertae. Assuming their filling factors to be 0.70 and 0.50 , we find: $\Delta v / v \sim 3.0 \times 10^{-3}$ and $\Delta v / v \sim 0.1$, respectively. The large shift obtained in the case of the subgiant in AR Lac is mainly the result of its large radius $R \simeq 3 R_{\odot}$, and cannot be regarded as a small perturbation in the sense of the Goldreich et al. (1991) model. In any case, this result suggests that surface magnetic effects may be significantly higher in subgiants than in dwarf stars of comparable activity level.

In the case of the active component of V471 Tau, for the mode with $n=20, l=0$, the magnetically induced perturbation is estimated to be $\approx 0.5 \mu \mathrm{Hz}$. It will not distort the asymptotic spectrum too much because it has a large separation of the peaks of $\approx 150 \mu \mathrm{Hz}$ and a small separation of $\approx 5 \mu \mathrm{Hz}$ (cf. Brown \& Gilliland 1994) ${ }^{1}$.

Activity cycles in very active stars are characterized by large variations of the spot filling factors up to $~ 30-40 \%$ (e.g., Rodonò et al. 1995; Lanza et al. 2001). It is not know whether the filling factor of the magnetic field outside spots, which is considered responsible for the frequency shift in the present

1 The dependence of the frequency shift on the degree $l$ is detectable in the solar case, solar activity signatures being well concentrated in latitude, but it is likely to be weak in very active stars with active regions widely spread in latitude and any clear signature of their regular migration along the cycle absent (cf., e.g., Hall 1991; Strassmeier \& Bartus 2000). 
model, is also subject to such large variations. It is likely that in very active stars the field filling factor is at a saturation level, thus the expected fluctuations of $f$ should be smaller, i.e., of the order of $5-10 \%$ at most. The time scales for such small changes may be quite short because sizeable changes of the spot pattern are usually observed on timescales of a few rotations. Therefore, it is likely that the frequency shift would fluctuate by $\sim 5-10 \%$ during the typical intervals along which asteroseismic data sets will be acquired (Brown \& Gilliland 1994). In other words, the spectral lines of the oscillation spectrum are expected to be broadened, in addition to finite mode lifetime, by the shift fluctuation due to surface activity. The contribution to the line width may be of the order of $50 \mathrm{nHz}$, in the case of the $n=20, l=0$ mode in the spectrum of V471 Tau we considered above. However, it may be considerably reduced in the case of lower frequency $p$-modes, because such a line broadening should scale with the square of the frequency (cf. Eq. (20) of Goldreich et al. 1991). Larger broadenings are expected for active subgiants and this may significantly limit the precision of the $p$-mode frequency measurements.

In the case of distant stars, only a limited number of $l$ and $m$ values are accessible by the observations, therefore it is not possible to pursue the expansion indicated in Eq. (1) beyond $N=2$ or 3 . Magnetic activity is expected to influence the even coefficients that in our case reduce to $a_{2}$, which sample the quadrupolar component of the magnetic perturbation, i.e., that described by the second-order Legendre polynomial $P_{2}$. The perturbation of the $a_{2}$ coefficient due to surface magnetic fields may be estimated according to, e.g., Dziembowski \& Goode (1991). They showed that, for a given surface distribution of the magnetic flux, the perturbation increases proportionally to the $P_{2}$ component of the latitudinal distribution of the filling factor. In very active stars, the time variation of the surface distribution of the field is likely to induce a variable $a_{2}$ coefficient with a characteristic time scale ruled by the fluctuations of the surface filling factor and the latitudinal migration of the magnetic flux, i.e., between 100-1000 days (cf., e.g., Donati 1999). Assuming that the typical filling factor fluctuations are of the order of $10 \%$, the expected amplitude for the variations of the measured $a_{2}$ coefficients in V471 Tau are of the order of $\approx 1 \mu \mathrm{Hz}$.

Subgiants and giant components in classical and long-term RS CVn systems are expected to show larger magnetically induced perturbations of their oscillation spectra, which makes more difficult the extraction of the asteroseismic information. Therefore, we focus our discussion on short-period RS CVn systems.

\section{Frequency changes expected by quadrupole moment changes}

The models proposed by Applegate (1992) and Lanza et al. (1998a) assume that the quadrupole moment of an active star changes along the activity cycle in response to the variation of the internal centrifugal and Lorentz forces. Consequently, three specific sources of $p$-mode frequency changes can be identified: a) the modification of the internal stratification due to the varying centrifugal and Lorentz forces; b) the changes of the
Table 1. Stellar parameter of the active star model.

\begin{tabular}{lc}
\hline \hline Mass $M$ & $0.700 M_{\odot}$ \\
Radius $R$ & $0.652 R_{\odot}$ \\
Luminosity $L$ & $0.177 L_{\odot}$ \\
$T_{\text {eff }}$ & $4640 \mathrm{~K}$ \\
$r_{\mathrm{c}} / R$ & 0.678 \\
$m_{\mathrm{c}} / M$ & 0.899 \\
\hline
\end{tabular}

propagation speed of the acoustic waves due to the variation of the internal magnetic field; c) the modification of the internal rotation, which produces a change of the advection term in the momentum equation. The first two sources produce effects that are insensitive to the sign of the azimuthal order $m$ because they cannot distinguish between the sense of propagation of a wave along the equator. Therefore, they affect only the even coefficients $a_{2 i}$ in the splitting expansion. Their effect on the $a_{2}$ coefficient can be easily estimated from the theory developed by Gough \& Thompson (1990). Typically, the $a_{2}$ produced by stellar rotation is of the order of $\left(\frac{\Omega^{2} R^{3}}{G M}\right) v \sim\left(10^{-3}-2 \times 10^{-2}\right) \times v$ for RS CVn and Algol systems, where $\Omega$ is the angular velocity of rotation and $G$ the gravitational constant. The variation of the splitting coefficients, that is produced by the relative variation $\Delta \Omega / \Omega$ of the angular velocity of the outer shell of the stellar convection zone, is of the order:

$\frac{\Delta a_{2}}{a_{2}} \sim 2\left(\frac{\Delta \Omega}{\Omega}\right)$.

This implies a change of the order of $\Delta a_{2} \sim 0.01-0.05 \mu \mathrm{Hz}$ for $\Delta \Omega / \Omega \sim 0.01$ and, therefore, it is not detectable, being about 10 times smaller than the fluctuations of the $a_{2}$ coefficients due to magnetic activity (cf. Sect. 2). Similar results are obtained when the perturbations to the inner density or sound speed by a strong internal magnetic field are considered.

The only change that may give rise to observable effects is related to the variation of the advection term in the momentum equation due to rotation. It manifests in the variation of the odd $a_{2 i+1}$ coefficients, in particular of the $a_{1}$ coefficients (cf., e.g., Gough \& Thompson 1990).

In order to correlate the variation of the orbital period with the internal rotation change and the variation of the $a_{1}$ coefficients, we assume a specific realization of Applegate's model. We consider an active star of mass $0.7 M_{\odot}$ in a close binary system with a companion of the same mass on a 12-hr orbit, which represents a typical short-period RS CVn system. A simple structure model for the active star is computed using the code by Hansen \& Kawaler (1994) assuming a homogeneous ZAMS structure with $X=0.74$ and $Y=0.24$. The basic parameters of our model star are listed in Table 1 , where $r_{\mathrm{c}} / R$ is the fractional radius at the base of the convection zone and $m_{\mathrm{c}} / M$ the fractional mass at the base of the convection zone. A comparison with the more accurate models by VandenBerg et al. (1983) shows that our structure quantities are correct within $\sim 10 \%$.

In order to produce a quadrupole moment variation, we consider a time-dependent differential rotation with an angular velocity $\Omega=\Omega(r, t)$. At the beginning of the activity cycle, at 


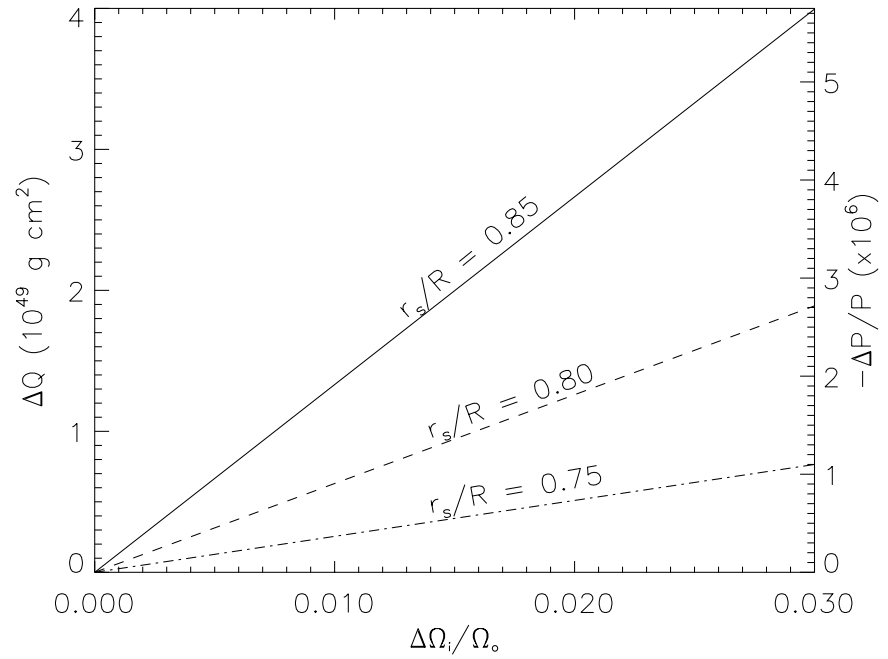

Fig. 1. The quadrupole moment changes $\Delta Q$ (scale on the left) and the corresponding orbital period variation $\Delta P / P$ (scale on the right) versus the relative change of the inner shell angular velocity $\Delta \Omega_{\mathrm{i}} / \Omega_{0}$ for three different values of the ratio $r_{\mathrm{s}} / R$ (as labelled).

$t=t_{0}$, the rotation is rigid with $\Omega\left(r, t_{0}\right)=\Omega_{0}$, where $\Omega_{0}$ is equal to the angular velocity of the orbital motion. Half a cycle later, at time $t_{1}$, the internal angular velocity is given by:

$\Omega\left(r, t_{1}\right)= \begin{cases}\Omega_{0} & r<r_{\mathrm{c}} \\ \Omega_{0}+\Delta \Omega_{\mathrm{i}} & r_{\mathrm{c}}<r<r_{\mathrm{s}} \\ \Omega_{0}-\Delta \Omega_{\mathrm{e}} & r_{\mathrm{s}}<r<R\end{cases}$

where $r_{\mathrm{s}}$ is the outer radius of the inner shell participating in the exchange of angular momentum and $\Delta \Omega_{\mathrm{i}}$ and $\Delta \Omega_{\mathrm{e}}$ denote the variation of the angular velocity in the inner and outer shells, respectively. They are constrained by total angular momentum conservation, that is:

$$
I_{\mathrm{i}} \Delta \Omega_{\mathrm{i}}+I_{\mathrm{e}} \Delta \Omega_{\mathrm{e}}=0
$$

where $I_{\mathrm{i}}$ and $I_{\mathrm{e}}$ are the moments of inertia of the inner and outer shells, respectively.

We computed the quadrupole moment change $\Delta Q$ between times $t_{0}$ and $t_{1}$ as a function of $\Delta \Omega_{\mathrm{i}} / \Omega_{0}$ for three different values of $r_{\mathrm{s}} / R$ by the method outlined in Goldreich \& Schubert (1968) (see also Paternò et al. 1996). The corresponding orbital period changes were evaluated for our model binary and are plotted together with $\Delta Q$ in Fig. 1. We notice that the quadrupole moment increases with the angular momentum of the inner shell because it is significantly more massive than the outer shell and thus dominates the variation of $Q$. The sign of $\Delta P$ is negative because $P$ decreases when the quadrupole moment increases.

In order to compute the variation of the $p$-mode splitting produced by the assumed rotation changes, we considered modes with radial order $n=23,24$ and $1 \leq l \leq 3$, which have frequencies near the expected maximum of the power spectrum of our model star, according to Kjeldsen \& Bedding (1995). The frequencies and the eigenfunctions of the $p$-modes of the non-rotating star were computed using the pulsation code by Hansen \& Kawaler (1994). The mode frequencies and the $a_{1}$ splitting coefficients for rigid rotation with angular velocity $\Omega_{0}$ are listed in Table 2 .
Table 2. Frequency and rotational splitting of the considered $p$-modes for rigid rotation.

\begin{tabular}{cccc}
\hline \hline Radial Order & Degree & $\begin{array}{c}\text { Frequency } \\
(\mu \mathrm{Hz})\end{array}$ & $\begin{array}{c}a_{1} \\
(\mu \mathrm{Hz})\end{array}$ \\
\hline$n=24$ & $l=1$ & 5585 & 22.23 \\
$n=24$ & $l=2$ & 5679 & 22.16 \\
$n=23$ & $l=3$ & 5556 & 22.15 \\
\hline
\end{tabular}

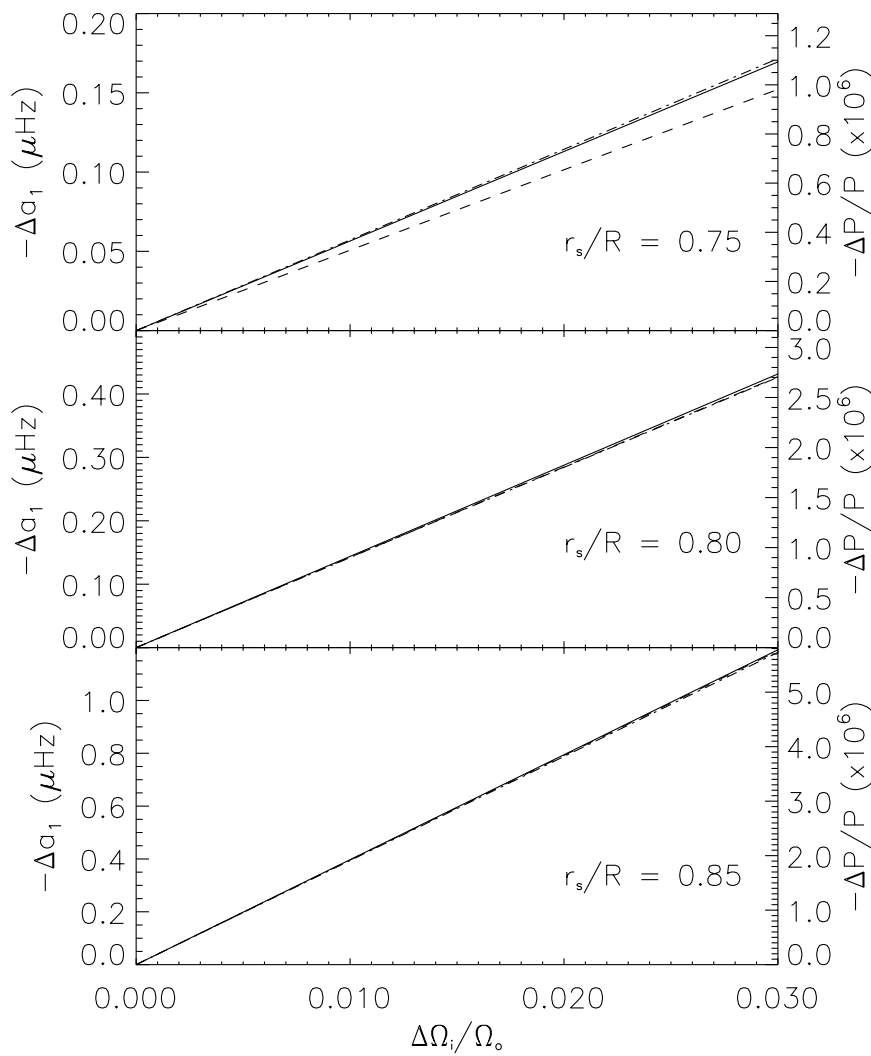

Fig. 2. The variation of the splitting coefficients $\Delta a_{1}$ for the three $p$-modes in Table 1 versus the relative angular velocity change in the inner shell of the convection zone $\Delta \Omega_{\mathrm{i}} / \Omega_{0}$. The splitting coefficients refer to modes with $n=24, l=1$ (solid line), $n=24, l=2$ (dashed line) and $n=23, l=3$ (dot-dashed line), respectively. The upper panel reports the results for $r_{\mathrm{s}} / R=0.75$, the middle one for $r_{\mathrm{s}} / R=0.80$ and the lower one for $r_{\mathrm{s}} / R=0.85$, respectively.

The variation of the $a_{1}$ coefficients was computed according to Brown et al. (1989). The results are reported in Fig. 2 as a function of the relative change of the internal angular velocity and for the three values of $r_{\mathrm{s}} / R$. It is interesting to note that our results are not affected by an internal latitudinal differential rotation profile, provided that it does not change with time. In fact, the $a_{1}$ coefficients are linear functions of the angular velocity profile (cf. Brown et al. 1989). The sizeable increase of the variation of the splitting coefficients $a_{1}$ with $r_{\mathrm{s}} / R$ is due to the increase of the angular velocity variation of the outer shell due to the steep increase of the ratio $I_{\mathrm{i}} / I_{\mathrm{e}}$ with $r_{\mathrm{s}} / R: I_{\mathrm{i}} / I_{\mathrm{e}}$ goes from 0.52 for $r_{\mathrm{s}} / R=0.75$ to 3.57 for $r_{\mathrm{s}} / R=0.85$. The $p$-mode splittings predominantly sample the angular velocity variations of the outer shell because the envelope of their rotational 
kernels scales approximately as $c_{\mathrm{s}}^{-1}$, where $c_{\mathrm{s}}$ is the sound speed (cf. Christensen-Dalsgaard 1988).

\section{Discussion and conclusions}

Our model for the quadrupole moment changes is based on the simple approach outlined by Applegate (1992) and allows a clear identification of the basic physical effects. In particular, the quadrupole moment changes arise mainly from the inner shell, whereas the splittings of the $p$-modes arise predominantly from the outer shell. A situation with $I_{\mathrm{i}} \gg I_{\mathrm{e}}$ would be ideal for maximizing the splitting variations, but it is unlikely to occur, considering the large kinetic energy change that is required (cf. Applegate 1992; Lanza \& Rodonò 1999).

If we adopt the models proposed by Lanza et al. (1998a) and Lanza \& Rodonò (1999), the splitting variations are reduced because a fraction of the quadrupole moment change is produced by the Lorentz force and not by a change of the internal rotation. However, the most likely situation is that in which the contributions of both effects are comparable, the expected splitting variations being reduced by about a factor of two. Moreover, the model proposed by Lanza et al. (1998a) predicts a cycle for the orbital period change with a length twice the spot activity cycle. The $a_{1}$ frequency splittings are expected to show the same period of the orbital period modulation, that is, twice the spot area cycle.

The $a_{1}$ coefficients can be derived from the observed frequencies in a multiplet by means of the formula:

$a_{1}(n, l)=\frac{v_{n, l, m}-v_{n, l,-m}}{2 m}$.

Given the presence of random errors, the actual values are derived by means of a best fit over the different azimuthal numbers $m$ of each mode. It is interesting to note that the contributions arising from the varying centrifugal and Lorentz forces cancel out in Eq. (6) because they depend on $|m|$ and the same is true also for the surface magnetic effects (cf. Sect. 2). The contributions from possible $a_{3}$ coefficients cancel out when time variations are considered, because the $a_{3}$ coefficients sample the latitudinal profile of the differential rotation, which is assumed to be constant in our model (cf. Brown et al. 1989).

The detection of the variation of the splitting coefficients requires a sufficiently high photometric accuracy and sufficiently long time series to resolve the individual frequencies. According to Kjeldsen \& Bedding (1995), the amplitude of the $p$-modes should approximately scale as $L / M$, which implies that the luminosity change expected for our model star is $\Delta L / L \approx 1.2 \times 10^{-6}$ at the peak of the $p$-mode spectrum for an isophotal wavelength $\lambda=550 \mathrm{~nm}$. A one-meter space-borne telescope may detect a single oscillation with that amplitude in an $m_{V}=10$ star from a continuous 50-60 day monitoring time (cf. Brown \& Gilliland 1994). The accuracy of the frequency determination is a function of the mode lifetime, of the ratio between the power level of the mode and the background noise and of the total observing time (Libbrecht 1992). Unfortunately, the first two quantities are unknown in the stellar case and they can be roughly estimated only on the basis of the solar analogy. Specifically, if we assume that the background noise due to convection scales as $L / M$ and that the mode lifetimes are comparable to those of the solar modes, an accuracy of $0.1 \mu \mathrm{Hz}$ may well be achieved in an observing time of $2-3 \mathrm{yr}$ (Libbrecht 1992). However, a significant source of noise could be related to magnetic activity, in particular to microflaring which might show timescales comparable with those of the $p$ modes. Multi-band simultaneous observations could be used to isolate the effects of microflaring because flares have a characteristic wavelength dependence in the optical band since their amplitude increases toward shorter wavelengths.

The broadening of the $p$-mode peaks due to surface magnetic activity may set an intrinsic limit to the accuracy of the frequencies. However, our estimates in Sect. 2 suggest that it should be at least 2-3 times smaller than the expected frequency changes near the peak of the power spectrum.

We conclude that the rotation changes predicted by Applegate's model may be detected by a long-term monitoring of an active star from a space-borne observatory. The variation of the frequency splittings can be correlated with the orbital period change allowing us to test the different versions of the model and to infer interesting information on the operation of the non-linear stellar dynamo. It is important to note that we are not proposing to measure the internal rotation of the star, for which the accuracy would be very low (cf. Goupil et al. 1996), but its time variations and to correlate them with an independent phenomenon, i.e., the orbital period change. The most suitable objects to which such a test can be applied are the very active dwarf stars in bright, short-period RS CVn systems, like V 471 Tau, RT And, CG Cyg, for which the orbital period changes can be monitored with rather good accuracy. We notice that the method is not restricted to eclipsing binaries because orbital period changes can be detected in spectroscopic binaries through long-term radial velocity monitoring (Frasca \& Lanza 2000).

The long time scales of the orbital period changes (from several years to a few decades), should require successive generations of space-borne observatories such as the planned COROT mission (e.g., Baglin et al. 2001) and the still under definition Eddington mission. The best observational strategy would be to observe the candidate system before and after a significant change of the orbital period had occured and that can be easily detected by ground-based long-term monitoring programs (cf., e.g., Rodonò et al. 2001). The precise determination of the splitting coefficients $a_{1}$ requires time series extending for at least 2-3 yr, which is within the expected lifetimes of the planned missions, although their observing strategy is to spend on average 5-6 months on each group of targets in the same field of view in order to maximize the number of stars observed. Therefore, our proposal demands a substantial modification of the planned mission strategy to be fulfilled, significantly reducing the number of targets in order to increase the time spent observing each of them and to achieve a frequency accuracy of $\sim 0.1 \mu \mathrm{Hz}$.

Acknowledgements. The authors wish to thank L. Paternò and M. P. Di Mauro for a critical reading of the manuscript and stimulating comments. They are also grateful to the Referee, C. Catala, for his 
careful reading and interesting comments. Research on stellar physics at Catania Astrophysical Observatory of the Istituto Nazionale di Astrofisica and at the Dept. of Physics and Astronomy of Catania University is funded by MIUR (Ministero dell'Istruzione, Università e Ricerca) and Regione Sicilia, whose financial support is gratefully acknowledged.

\section{References}

Applegate, J. H. 1992, ApJ, 385, 621

Baglin, A., Auvergne, M., Catala, C., Michel, E., \& COROT Team 2001, ESA-SP 464, 395

Brown, T. M., Christensen-Dalsgaard, J., Dziembowski, W. A., et al. 1989, ApJ, 343, 526

Brown, T. M., \& Gilliland, R. L. 1994, ARA\&A, 32, 37

Christensen-Dalsgaard, J. 1988, in Advances in Helio- and Asteroseismology, ed. J. Christensen-Dalsgaard, \& S. Frandsen (D. Reidel, Publ. Co., Dordrecht), 3

Christensen-Dalsgaard, J., Däppen, W., Dziembowski, W. A., \& Guzik, J. A. 2000, in Variable Stars as Essential Astrophysical Tools, ed. C. İbanoğlu (Kluwer Ac. Publ., Dordrecht), 59

Donati, J.-F. 1999, MNRAS, 302, 457

Dziembowski, W. A., \& Goode, Ph. R. 1991, ApJ, 376, 782

Eaton, J. A., Henry, G. W., \& Fekel, F. C. 1996, ApJ, 462, 888

Frasca, A., \& Lanza, A. F. 2000, A\&A, 356, 267

Goldreich, P., Murray, N., \& Willette, G. 1991, ApJ, 370, 752

Goldreich, P., \& Schubert, G. 1968, ApJ, 154, 1005

Gough, D. O., \& Thompson, M. J. 1990, MNRAS, 242, 25

Goupil, M.-J., Dziembowski, W. A., Goode, Ph. R., \& Michel, E. 1996, A\&A, 305, 487

Hall, D. S. 1989, Space Sci. Rev., 50, 219
Hall, D. S. 1991, in The Sun and cool stars: Activity, magnetism, dynamos, ed. I. Tuominen, D. Moss, \& G. Rüdiger (Springer-Verlag, Berlin), 353

Hansen, C. J., \& Kawaler, S. D. 1994, Stellar Interiors (SpringerVerlag, Berlin)

Henry, G. W., Eaton, J. A., Hamer, J., \& Hall, D. S. 1995, ApJS, 97, 513

Howe, R., Christensen-Dalsgaard, J., Hill, F., et al. 2000, Science, 287, 2456

Howe, R., Komm, R., \& Hill, F. 1999, ApJ, 524, 1084

Kjeldsen, H., \& Bedding, T. R. 1995, A\&A, 293, 87

Lanza, A. F., \& Rodonò, M. 1999, A\&A, 349, 887

Lanza, A. F., Catalano, S., Cutispoto, G., Pagano, I., \& Rodonò, M. 1998b, A\&A, 332, 541

Lanza, A. F., Rodonò, M., \& Rosner, R. 1998a, MNRAS, 296, 893

Lanza, A. F., Rodonò, M., Mazzola, L., \& Messina, S. 2001, A\&A, 376, 1011

Lanza, A. F., Catalano, S., Rodonò, M., et al. 2002, A\&A, 386, 583

Libbrecht, K. G. 1992, ApJ, 387, 712

Oláh, K., Kövari, Zs., Bartus, J., et al. 1997, A\&A, 321, 811

Paternò, L., Sofia, S., \& Di Mauro, M. P. 1996, A\&A, 314, 940

Rodonò, M., Lanza, A. F., \& Catalano, S. 1995, A\&A, 301, 75

Rodonò, M., Cutispoto, G., Lanza, A. F., \& Messina, S. 2001, AN, 322,333

Saar, S. H. 1996, Magnetodynamic Phenomena in the Solar Atmosphere, ed. Y. Uchida, T. Kosugi, \& H. S. Hudson (Kluwer Ac. Publ., Dordrecht), 367

Schou, J., Antia, H. M., Basu, S., et al. 1998, ApJ, 505, 390

Strassmeier, K. G., \& Bartus, J. 2000, A\&A, 354, 537

Tassoul, M. 1980, ApJS, 43, 469

VandenBerg, D. A., Hartwick, F. D. A., Dawson, P., \& Alexander, D. R. 1983, ApJ, 266, 747 